\title{
Hemodynamic Characteristics of Ruptured and Unruptured Multiple Aneurysms at Mirror and Ipsilateral Locations
}

\author{
(D) R. Doddasomayajula, (D)B.J. Chung, (DF. Mut, (D) C.M. Jimenez, (D)F. Hamzei-Sichani, (D) C.M. Putman, and (D).R. Cebral
}

\begin{abstract}
BACKGROUND AND PURPOSE: Different hemodynamic patterns have been associated with aneurysm rupture. The objective was to test whether hemodynamic characteristics of the ruptured aneurysm in patients with multiple aneurysms were different from those in unruptured aneurysms in the same patient.
\end{abstract}

MATERIALS AND METHODS: Twenty-four mirror and 58 ipsilateral multiple aneurysms with 1 ruptured and the others unruptured were studied. Computational fluid dynamics models were created from 3D angiographies. Case-control studies of mirror and ipsilateral aneurysms were performed with paired Wilcoxon tests.

RESULTS: In mirror pairs, the ruptured aneurysm had more oscillatory wall shear stress $(P=.007)$ than the unruptured one and tended to be more elongated (higher aspect ratio), though this trend achieved only marginal significance $(P=.03$, 1-sided test). In ipsilateral aneurysms, ruptured aneurysms had larger maximum wall shear $(P=.05)$, more concentrated $(P<.001)$ and oscillatory wall shear stress $(P<$ $.001)$, stronger $(P<.001)$ and more concentrated inflow jets $(P<.001)$, larger maximum velocity $(P<.001)$, and more complex flow patterns $(P<.001)$ compared with unruptured aneurysms. Additionally, ruptured aneurysms were larger $(P<.001)$ and more elongated $(P<.001)$ and had wider necks $(P<.001)$ and lower minimum wall shear stress $(P<.001)$ than unruptured aneurysms.

CONCLUSIONS: High wall shear stress oscillations and larger aspect ratios are associated with rupture in mirror aneurysms. Adverse flow conditions characterized by high and concentrated inflow jets; high, concentrated, and oscillatory wall shear stress; and strong, complex and unstable flow patterns are associated with rupture in ipsilateral multiple aneurysms. In multiple ipsilateral aneurysms, these unfavorable flow conditions are more likely to develop in larger, more elongated, more wide-necked, and more distal aneurysms.

ABBREVIATIONS: CORELEN = vortex core-line length; $\max =$ maximum; $\mathrm{min}=$ minimum; OSI = oscillatory shear index; PODENT = proper orthogonal decomposition entropy; WSS = wall shear stress

U unruptured cerebral aneurysms are diagnosed with increasing frequency, but despite the relatively low risk of rupture (estimated at $0.3 \%-3 \%{ }^{1}$ ) and because of high mortality and disability rates (estimated at $45 \%$ mortality in the first year ${ }^{2}$ and $64 \%$ disability of survivors ${ }^{3}$ ), patients often undergo preventive inter-

Received April 3, 2017; accepted after revision August 1.

From the Bioengineering Department (R.D., B.J.C., F.M., J.R.C.), George Mason University, Fairfax, Virginia; Neurosurgery Department (C.M.J.), University of Antioquia, Medellin, Colombia; Department of Neurosurgery (F.H.-S.), Mt. Sinai Medical Center, New York, New York; and Interventional Neuroradiology (C.M.P.), Inova Fairfax Hospital, Falls Church, Virginia.

This work was partially supported by the National Institutes of Health/National Institute of Neurological Disorders and Stroke grant No. R21NS094780.

Please address correspondence to Juan R. Cebral, PhD, Bioengineering Department, Volgenau School of Engineering, George Mason University, 4400 University Dr, MSN 2A1, Fairfax, VA 22030; e-mail: jcebral@gmu.edu

-- Indicates open access to non-subscribers at www.ajnr.org

三 Indicates article with supplemental on-line appendix and tables.

http://dx.doi.org/10.3174/ajnr.A5397 vention, which is not without risk of complications (estimated at $10 \%-14 \%$ combining perioperative morbidity and mortality ${ }^{4}$ ). Therefore, it is imperative to properly select patients, avoiding unnecessary and relatively risky procedures. Selection is particularly important in patients with multiple aneurysms (about 15\%$35 \%$ of all patients with cerebral aneurysms ${ }^{5,6}$ ) because in these cases, clinicians need to decide whether to treat each of the lesions, which may require multiple interventions, depending on accessibility and treatment technique (surgical or endovascular). ${ }^{7}$

The process underlying the rupture of a cerebral aneurysm is highly complex, and the principal factors leading to this event are largely unknown. ${ }^{1,4,8}$ Numerous features have been investigated as possible rupture risk factors: location, size, sex, age, family history, smoking, alcohol, and hypertension. ${ }^{9}$ Size and location have been identified as the most important aneurysm-specific predictors of rupture in both single and multiple aneurysms. ${ }^{10}$ However, it is wellknown that small aneurysms do rupture, including in cases of multiple aneurysms. ${ }^{6}$ Therefore, it is important to identify additional 
independent characteristics to improve the risk assessment of patients with multiple aneurysms. Among all the factors involved, the hemodynamics of the lesion are thought to be one of the fundamental players, ${ }^{11}$ interacting with the wall biology, which, in turn, drives the degradation of the wall structure and its mechanical strength.

Multiple aneurysms, in addition to being clinically very important and challenging, offer the unique opportunity for comparing ruptured (cases) and unruptured (controls) aneurysms while perfectly controlling for patient-related confounding factors such as sex, age, family history, previous hemorrhage, smoking, alcohol, drugs, and hypertension. Thus, the objective of this study was to test whether hemodynamic characteristics of the ruptured aneurysm in patients with multiple aneurysms were different from those of unruptured aneurysms in the same patient. This information is important not only to identify hemodynamic factors that could be used to assess which aneurysms are at higher risk of progressing toward rupture but also to better understand the mechanisms that drive the progressive degradation of the wall and ultimately result in aneurysm rupture.

\section{MATERIALS AND METHODS Image and Patient Data}

We have developed a data base of cerebral aneurysms imaged with 3D rotational angiography, mainly from Inova Fairfax Hospital (Northern Virginia), Mt. Sinai Medical Center (New York), and the Mayo Clinic (Rochester, Minnesota). This data base contains 3D rotational angiography images along with basic information, including aneurysm rupture status and location and size of $>2000$ cerebral aneurysms. The data have been anonymized; and the study was approved by the George Mason University institutional review board. For this study, 2 subsets of multiple aneurysms were considered, as detailed below.

\section{Mirror Aneurysms}

The first subset included mirror aneurysm pairs - that is, 2 aneurysms at the same anatomic location on each side. Furthermore, only mirror pairs with 1 ruptured and the other unruptured were considered. Forty-eight mirror aneurysms (24 ruptured and 24 corresponding contralateral unruptured ones) in 24 patients ( 21 [87.5\%] women, 3 [12.5\%] men; mean age, $52.9 \pm 13.3$ years; age range, $23-81$ years) were included in the study. Of the 24 pairs, 7 (29\%) were middle cerebral artery bifurcation aneurysms, while the remainder $(17,71 \%)$ were sidewall or lateral aneurysm pairs.

\section{Ipsilateral Multiple Aneurysms}

The second subset included multiple ipsilateral aneurysms - that is, multiple aneurysms along the same arterial tree. Again, only cases with 1 ruptured and $\geq 1$ unruptured aneurysm on the same arterial tree were considered. There were 58 patients (44 [76\%] women, 13 men [22.4\%], and 1 unknown; mean age, $58 \pm 14.7$ years; age range, $28-88$ years). This subset included 144 aneurysms, including 58 ruptured and 86 unruptured ones. There were 97 bifurcation (67\%), and 47 lateral (33\%) aneurysms.

\section{Vascular and Blood Flow Modeling}

Image-based computational fluid dynamics models of all 192 aneurysms (48 mirror and 144 ipsilateral aneurysms) were con- structed from the corresponding 3D rotational angiography images using previously described methods. ${ }^{12,13}$ For mirror pairs, 2 vascular models were independently constructed for the left and right sides; while for the ipsilateral cases, a single model, including all ipsilateral aneurysms, was created. Numeric simulations were performed under pulsatile flow for 2 cardiac cycles using a solver developed in-house. ${ }^{14}$ Blood was assumed to have Newtonian viscosity, and vessel wall compliance was neglected. The inflow conditions were derived from a phase-contrast MR imaging scan of a healthy subject that was scaled with the inflow vessel crosssectional area. ${ }^{15}$ Fully developed velocity profiles were prescribed at the inlets, while outflow boundary conditions were selected to produce flow divisions consistent with the Murray principle of minimal work. Results from the second simulated cardiac cycle were analyzed. Different aspects of the aneurysmal hemodynamic environment, including the wall shear stress distribution, the inflow jet, and the intra-aneurysmal flow pattern, were studied. Different variables or metrics were defined to capture different characteristics of the flow. ${ }^{16,17} \mathrm{~A}$ few additional geometric variables were computed from the reconstructed vascular models. The variables considered (On-line Appendix) are listed in On-line Tables 1-4. For ipsilateral aneurysms, an extra variable that assigns an increasing value for more distal aneurysms (thus called the "distality coordinate") was introduced (On-line Table 5).

\section{Data Analysis}

Matched case-control studies were performed for both mirror and ipsilateral aneurysms.

\section{Mirror Aneurysms}

The hemodynamic (and geometric) characteristics of ruptured (cases) and unruptured (controls) aneurysms in mirror pairs were compared with a 2-sided paired Wilcoxon test. Differences were considered significant if $P<.05$. By considering case-control pairs of the same patient, all patient-specific characteristics (eg, sex, age, comorbidities, habits, genetics, and so forth) were matched. Furthermore, in mirror pairs, aneurysm location was also matched by definition. The flow rate in the parent artery was not matched because 1 aneurysm is fed from 1 internal carotid artery, while the other is fed from the contralateral ICA.

\section{Ipsilateral Multiple Aneurysms}

Similarly, hemodynamic (and geometric) characteristics of ruptured (cases) and unruptured (controls) ipsilateral aneurysms were compared using a 2-sided paired Wilcoxon test. However, some patients had 1 ruptured aneurysm and $>1$ unruptured aneurysm (multiple controls). Therefore, in these cases, the ruptured aneurysm was paired with a randomly selected unruptured aneurysm of the same patient, thus making all aneurysm pairs independent. The process was repeated 100 times, and the mean and maximum $P$ values were calculated. If the maximum $P$ value of all 100 tests was $<.05$, the difference between ruptured and unruptured groups was considered statistically significant. If the mean $P$ value was $<.05$ but the maximum was not, the difference was considered marginally significant. Repeating the random selections 100 or 200 times did not show noticeable differences. Therefore, 100 repetitions were deemed sufficient. 
Hemodynamic and geometric characteristics of ruptured and unruptured mirror and multiple ipsilateral aneurysms ${ }^{\mathrm{a}}$

\begin{tabular}{|c|c|c|c|c|c|c|c|}
\hline \multirow[b]{2}{*}{ Variable } & \multicolumn{3}{|c|}{ Mirror Aneurysms } & \multicolumn{4}{|c|}{ Ipsilateral Aneurysms } \\
\hline & Unruptured & Ruptured & $P$ Value & Unruptured & Ruptured & Pmean & $P \max$ \\
\hline WSSmin & $0.6 \pm 0.9$ & $0.4 \pm 0.6$ & .24 & $1.3 \pm 2.0$ & $0.4 \pm 0.8$ & $<.001^{\mathrm{b}}$ & $<.001^{\mathrm{b}}$ \\
\hline WSSmax & $285.3 \pm 216.1$ & $269.5 \pm 183.1$ & 1.00 & $269.6 \pm 324.5$ & $784.9 \pm 2730.4$ & $.006^{\mathrm{b}}$ & $.05^{\mathrm{b}}$ \\
\hline WSSmean & $28.1 \pm 26.8$ & $22.3 \pm 18.6$ & .49 & $29.3 \pm 35.5$ & $27.1 \pm 36.8$ & .28 & .81 \\
\hline WSSnorm & $0.53 \pm 0.40$ & $0.44 \pm 0.26$ & .30 & $0.60 \pm 0.39$ & $0.50 \pm 0.32$ & .12 & .43 \\
\hline $\mathrm{SCl}$ & $5.48 \pm 10.87$ & $5.54 \pm 4.53$ & .21 & $3.30 \pm 3.95$ & $6.74 \pm 7.20$ & $<.001^{\mathrm{b}}$ & $<.001^{\mathrm{b}}$ \\
\hline LSA & $51.6 \pm 33.0$ & $50.9 \pm 33.4$ & .81 & $48.7 \pm 32.4$ & $49.6 \pm 32.0$ & .72 & 1.00 \\
\hline OSImax & $0.243 \pm 0.123$ & $0.334 \pm 0.115$ & $.008^{\mathrm{b}}$ & $0.181 \pm 0.134$ & $0.332 \pm 0.103$ & $<.001^{\mathrm{b}}$ & $<.001^{\mathrm{b}}$ \\
\hline OSImean & $0.012 \pm 0.010$ & $0.014 \pm 0.010$ & .55 & $0.009 \pm 0.011$ & $0.014 \pm 0.010$ & $.005^{b}$ & .06 \\
\hline $\mathrm{Q}$ & $0.80 \pm 1.15$ & $0.72 \pm 0.85$ & .79 & $0.23 \pm 0.29$ & $0.70 \pm 0.62$ & $<.001^{\mathrm{b}}$ & $<.001^{b}$ \\
\hline $\mathrm{ICl}$ & $0.72 \pm 0.89$ & $0.82 \pm 0.80$ & .66 & $0.24 \pm 0.43$ & $0.68 \pm 0.57$ & $<.001^{\mathrm{b}}$ & $<.001^{\mathrm{b}}$ \\
\hline Vmax & $82.3 \pm 62.5$ & $80.8 \pm 44.4$ & .88 & $61.9 \pm 63.1$ & $98.2 \pm 81.0$ & $<.001^{\mathrm{b}}$ & $<.001^{\mathrm{b}}$ \\
\hline VE & $11.8 \pm 10.1$ & $10.1 \pm 6.8$ & .77 & $9.1 \pm 8.9$ & $10.9 \pm 9.4$ & .29 & .70 \\
\hline SR & $259.6 \pm 192.3$ & $229.2 \pm 159.8$ & .68 & $339.6 \pm 305.0$ & $246.1 \pm 236.4$ & $.03^{b}$ & .18 \\
\hline VO & $363.1 \pm 284.9$ & $319.1 \pm 225.8$ & .70 & $445.7 \pm 421.7$ & $346.1 \pm 345.6$ & .09 & .38 \\
\hline CORELEN & $1.481 \pm 2.014$ & $2.501 \pm 3.159$ & .17 & $0.376 \pm 0.557$ & $2.226 \pm 2.144$ & $<.001^{\mathrm{b}}$ & $<.001^{\mathrm{b}}$ \\
\hline PODENT & $0.167 \pm 0.136$ & $0.175 \pm 0.074$ & .33 & $0.137 \pm 0.121$ & $0.192 \pm 0.127$ & $.009^{b}$ & .08 \\
\hline Asize & $0.594 \pm 0.331$ & $0.713 \pm 0.361$ & .14 & $0.365 \pm 0.185$ & $0.726 \pm 0.282$ & $<.001^{\mathrm{b}}$ & $<.001^{b}$ \\
\hline Nsize & $0.412 \pm 0.185$ & $0.422 \pm 0.147$ & .75 & $0.306 \pm 0.127$ & $0.423 \pm 0.136$ & $<.001^{\mathrm{b}}$ & $<.001^{\mathrm{b}}$ \\
\hline AR & $0.956 \pm 0.755$ & $1.176 \pm 0.551$ & .06 & $0.669 \pm 0.408$ & $1.198 \pm 0.609$ & $<.001^{\mathrm{b}}$ & $<.001^{\mathrm{b}}$ \\
\hline Distality & - & - & - & $0.444 \pm 0.345$ & $0.612 \pm 0.321$ & $<.008^{\mathrm{b}}$ & $.04^{b}$ \\
\hline
\end{tabular}

Note:- LSA indicates percentage area under low WSS; SCl, shear concentration index; Vmax, maximum flow velocity; Q, aneurysm flow rate; ICl, inflow concentration index; norm, normalized; Asize, aneurysm maximum size; Nsize, neck maximum size; AR, aspect ratio ( $\leq$ depth/neck size); VO, mean aneurysm vorticity; SR, shear rates; VE, mean aneurysm velocity; Distality, coordinate of vessel segment along the arterial tree.

${ }^{a}$ Values are given as mean \pm SD. $P$ values correspond to univariate paired Wilcoxon rank sum tests. For ipsilateral aneurysms, mean and maximum $P$ values of 100 randomly selected ruptured-unruptured pairs are given.

b Significant difference $(P<.05)$.

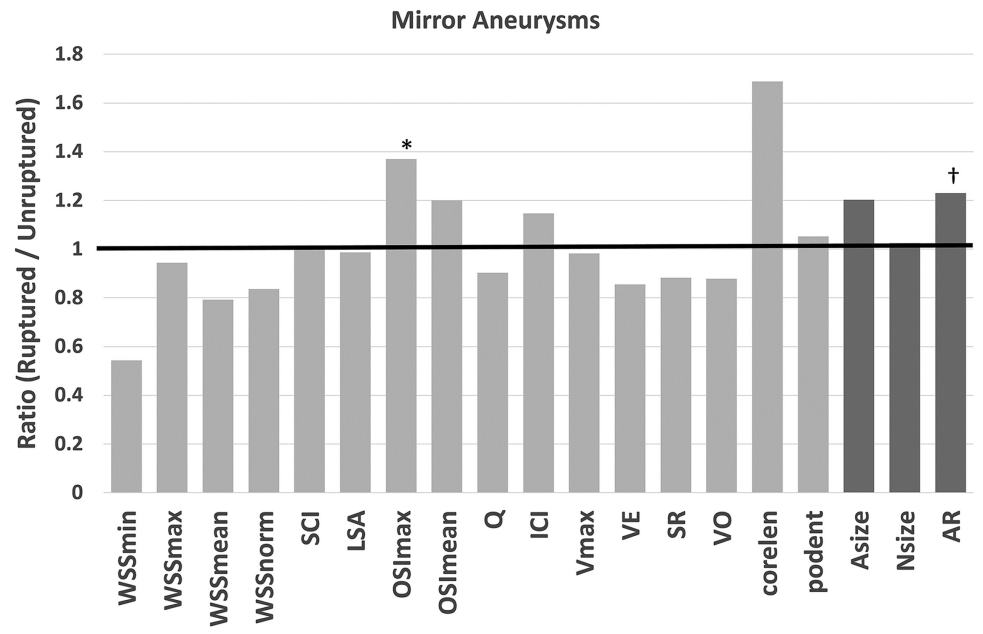

FIG 1. Ratios of mean values of hemodynamic and geometric variables of ruptured over unruptured mirror aneurysms. Statistically significant differences $(P<.05)$ are indicated with an asterisk, and marginally significant differences ( $P<.05$ on 1-sided test), with a dagger. LSA indicates percentage area under low WSS; $\mathrm{SCl}$, shear concentration index; Vmax, maximum flow velocity; $\mathrm{Q}$, aneurysm flow rate; ICI, inflow concentration index; norm, normal; Asize, aneurysm maximum size; Nsize, neck maximum size; AR, aspect ratio ( $\leq$ depth/neck size); VO, mean aneurysm vorticity; SR, shear rates; VE, mean aneurysm velocity.

\section{RESULTS}

Comparisons of the mean values of hemodynamic (and geometric) variables between ruptured and unruptured aneurysms in both mirror pairs and ipsilateral multiple aneurysms are presented in the Table. This Table lists the mean value \pm the SD of each variable over the corresponding matched ruptured and unruptured groups. In the mirror cases, the $P$ value corresponds to the paired Wilcoxon test. In ipsilateral aneurysms, the mean and maximum $(P \max ) P$ values over 100 random pairings are given. Statistically significant differences $(P<.05)$ are indicated with a superscript $b$.
The findings for mirror aneurysms are summarized in Fig 1. This figure presents the ratio of the mean values of hemodynamic (and geometric) variables of ruptured over unruptured aneurysms. In mirror aneurysms, the ruptured aneurysms had more oscillatory wall shear stress distributions (higher oscillatory shear index maximum [OSImax], $P=.007$ ) than unruptured aneurysms. Additionally, ruptured aneurysms were more elongated (ie, higher aspect ratio, $P=.06$ ), though this trend achieved only marginal significance $(P=.03$ for a 1 -sided test, $P=.06$ for a 2 -sided test). The inflow rate imposed as boundary conditions based on the parent artery diameter was not significantly different between ruptured and unruptured aneurysms $(P=.12)$.

The results for ipsilateral aneurysms are summarized in Fig 2. The error bars indicate the variability of the ratio of the mean values of ruptured over unruptured aneurysms for 100 random pairings. In multiple ipsilateral aneurysms, ruptured aneurysms had larger maximum WSS (WSSmax, $P \max =.05$ ), more concentrated and oscillatory WSS distributions (shear concentration index, $P \max <.001$; OSImax, $P \max <.001$; OSImean, mean $P=.005)$, stronger and more concentrated inflow jets (aneurysm flow rate, $P \max <.001$; inflow concentration index, $P \max <.001$ ), a larger maximum flow velocity $(P \max <.001)$, and more complex and unstable flow 


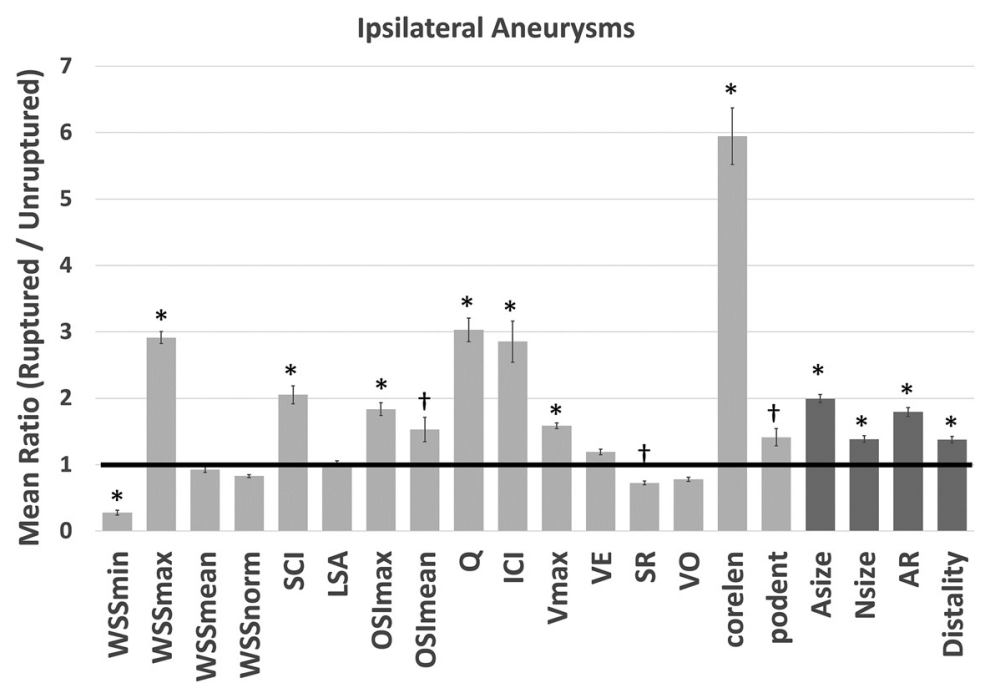

FIG 2. Ratios of mean values of hemodynamic and geometric variables of ruptured over unruptured ipsilateral aneurysms. Error bars indicate variability of the mean ratios over 100 random selections of ruptured-unruptured multiple aneurysm pairs. Statistically significant differences $(P \max <.05)$ are indicated with an asterisk, and marginally significant differences (mean $P=.05$ but $P \max >.05$ ), with a dagger. LSA indicates percentage area under low WSS; SCI, shear concentration index; $V \max$, maximum flow velocity; $\mathrm{Q}$, aneurysm flow rate; $\mathrm{ICl}$, inflow concentration index; norm, normal; Asize, aneurysm maximum size; Nsize, neck maximum size; AR, aspect ratio ( $\leq$ depth/neck size); VO, mean aneurysm vorticity; SR, shear rates; VE, mean aneurysm velocity.

patterns (vortex core-line length [CORELEN], Pmax $<.001$; proper orthogonal decomposition entropy [PODENT], mean $P=$ .009) compared with unruptured aneurysms. Additionally, ruptured aneurysms had lower minimum WSS (WSSmin, Pmax $<$ .001 ) and shear rates (mean $P=.03$ ) than unruptured aneurysms. Geometrically, ruptured ipsilateral aneurysms were larger (aneurysm maximum size, $P \max <.001$ ), had wider necks (neck maximum size, $P \max <.001$ ), were more elongated (aspect ratio, $P \max <.001)$, and were located more distally $(P \max =.04)$ than unruptured aneurysms.

\section{DISCUSSION}

Between $10 \%$ and $33 \%$ of patients with subarachnoid hemorrhage have multiple aneurysms, and the approach to these patients becomes complex considering that it is difficult to unequivocally identify the ruptured aneurysm when angiography reveals multiple aneurysms. ${ }^{18}$ On the other hand, whether the intervention of patients with multiple aneurysms should be directed to only the ones that bled or to all the lesions found on angiography has been long debated. ${ }^{19}$ If none have ruptured, the one at greatest risk of rupture should be identified. Localization and size have traditionally been the 2 variables that have defined the risk of rupture in multiple cerebral aneurysms; however, some other geometric and hemodynamic variables have emerged. ${ }^{20}$

The current study took advantage of multiple aneurysms (1 ruptured and $\geq 1$ unruptured in the same patient) offering a unique opportunity to compare ruptured and unruptured aneurysms while controlling for all patient-specific characteristics, eliminating confounding factors when each patient functions as his or her own internal control because all paired aneurysms are in the same patient. Some authors have argued that otherwise, patient-related confounding variables such as history of smoking, history of hypertension, or even genetic predisposition are diffi- cult or even almost impossible to control for or eliminate. ${ }^{20}$ Furthermore, the study was subdivided into the analysis of mirror pairs, in which aneurysm location but not inflow conditions were controlled; and ipsilateral multiple aneurysms, in which inflow conditions but not location were controlled. The objective of these comparisons was to identify aneurysm-specific characteristics that are independent of patient-specific characteristics and can discriminate ruptured and unruptured aneurysms. These aneurysm-specific characteristics could complement patient-specific risk factors in identifying aneurysms at higher risk of rupture. The study focused on aneurysm hemodynamic characteristics because it is thought that adverse hemodynamic conditions could predispose aneurysm walls to further degradation and eventual rupture. ${ }^{21,22}$

In our study, ruptured aneurysms in mirror pairs had more oscillatory WSS distributions (characterized by larger maximum OSI) and were more elongated (ie, having a marginally larger aspect ratio) than the corresponding unruptured aneurysms. An example of a mirror pair is presented in Fig 3. In a previous study, Fan et $\mathrm{al}^{23}$ analyzed 16 mirror bifurcation aneurysm pairs and found that ruptured aneurysms were larger (larger size and size ratio), were more elongated (larger aspect ratio and height-to-width ratio), and had lower mean WSS and larger area under low WSS. They also observed that ruptured aneurysms tended to have more complex and unstable flow patterns, but these results were not statistically significant. They also noted that the OSI might not be different in their sample because they excluded sidewall aneurysms. Likewise, in our sample, ruptured aneurysms tended to have lower mean WSS and more complex (larger CORELEN) flow patterns, but these results did not achieve statistical significance. However, the area under low WSS was not different in our study. In another study, Huang et $\mathrm{al}^{24}$ analyzed 63 mirror pairs imaged with CTA and found that ruptured aneurysms were larger (larger size, size ratio, area ratio), were more elongated (larger aspect ratio), and had more irregular walls than unruptured aneurysms. In our study, ruptured aneurysms were also more elongated than unruptured aneurysms, but they were not larger than the unruptured ones.

Our results indicate that in ipsilateral multiple aneurysms, the ruptured aneurysm tends to have hostile flow conditions characterized by higher and more concentrated inflow jets (larger aneurysm inflow rate and inflow concentration index); and higher, more concentrated, and more oscillatory WSS distributions (larger WSSmax, shear concentration index, and OSImax), with lower minimum WSS and stronger, more complex and unstable flow patterns (larger maximum flow velocity, CORELEN, and PODENT) with a lower shear rate, compared with unruptured aneurysms of the same patient. Because the aneurysms considered 

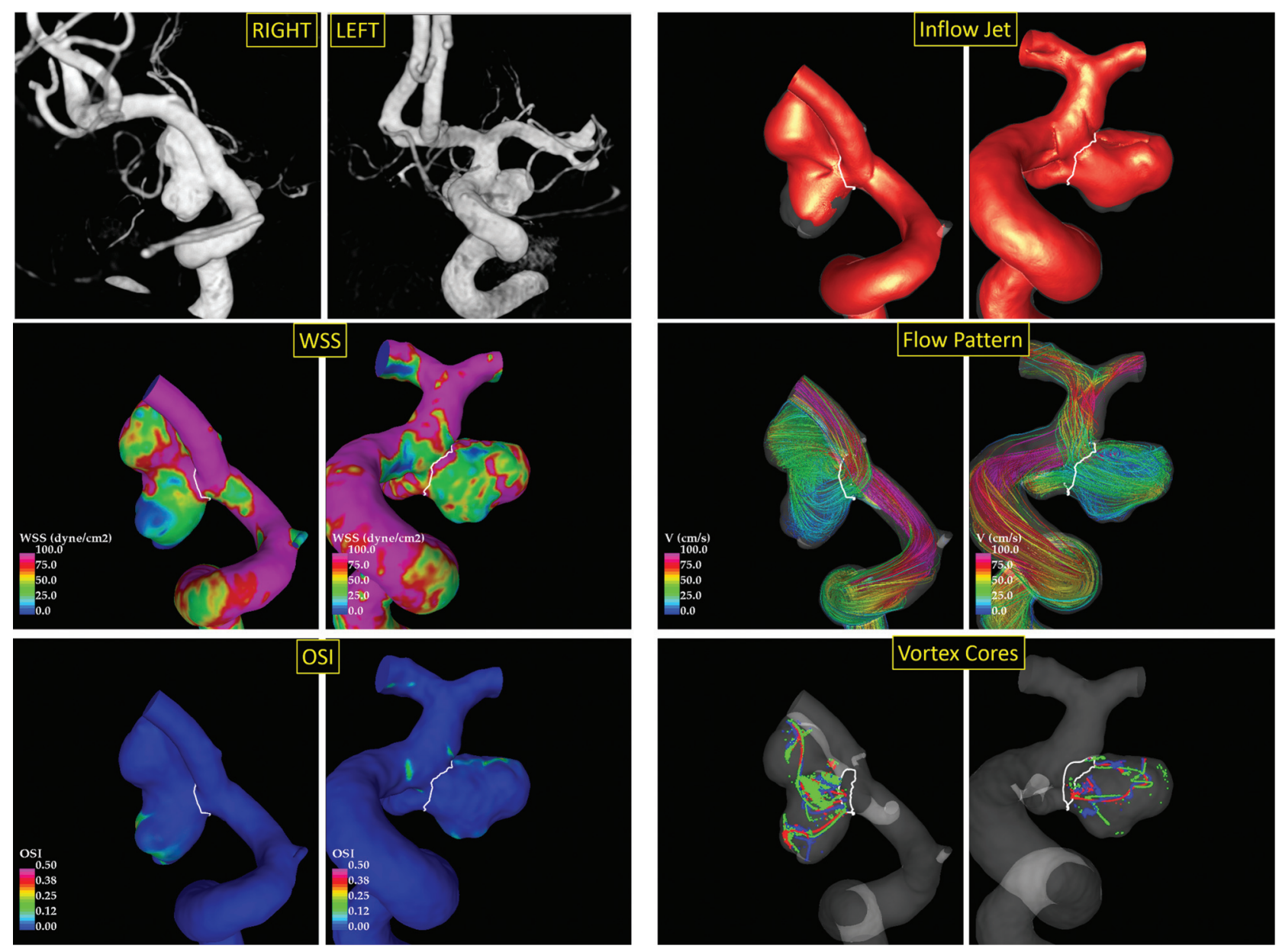

FIG 3. Example of ruptured (right posterior communicating artery aneurysm, left column) and unruptured (right posterior communicating artery aneurysm, right column) mirror aneurysm pairs. The Left panel shows from top to bottom: 3D rotational angiography images, WSS distributions, and OSI distributions. The right panel shows from top to bottom: inflow jets, flow patterns, and vortex core lines at 4 times during the cardiac cycle.

here occur on the same arterial tree in the same patient, these findings are independent of the patient-specific flow conditions. Additionally, it was found that ruptured aneurysms tended to be larger, with wider necks, and more elongated than unruptured ipsilateral aneurysms. Most interesting, in general, the ruptured aneurysms tended to be more distal than unruptured aneurysms. Examples of ipsilateral multiple aneurysms illustrating these flow conditions are presented in Fig 4. In a previous study, Zhang et $\mathrm{al}^{25}$ analyzed 20 ipsilateral aneurysm pairs and found that ruptured aneurysms were more irregular and elongated and had lower minimum WSS and larger areas under low WSS than unruptured aneurysms. Most interesting, in our study, the minimum WSS was also lower in ruptured than in unruptured aneurysms, but the area under low WSS was not different. Zhang et al reported no difference in bleeding sites of ipsilateral aneurysms, while Jou et $\mathrm{al}^{26}$ speculated that the proximal aneurysm in tandem serial ipsilateral aneurysms may have a higher rupture risk based on their analysis of 4 serial pairs. In contrast, our data suggest that ruptured aneurysms tended to be more distal than the unruptured aneurysms in the same patient.

The adverse flow conditions described above have been previously shown to be associated with aneurysm wall inflammation, which itself is associated with aneurysm rupture, ${ }^{27}$ wall weaken- ing and stiffening characteristic of vulnerable walls, ${ }^{28,29}$ and damaged collagen architectures. ${ }^{30}$ Additionally, these flow conditions have been associated with aneurysm rupture. ${ }^{31}$ Our results do not contradict reports that have associated low normalized WSS and large area under low WSS with rupture, ${ }^{32}$ though in our sample, normalized WSS and area under low WSS were not significantly different between ruptured and unruptured aneurysms in the same patients. What may constitute a hostile flow environment is a heterogeneous, oscillatory WSS distribution with focal elevations of WSS and large regions of low WSS produced by strong concentrated inflow jets that create complex unstable intrasaccular flow structures. The exact mechanisms that cause wall inflammation and rupture under high-flow conditions are still uncertain. However, this study provides valuable information to consider in future studies to understand the connection between the local flow conditions and the structure and strength of the wall.

Location has been previously identified as a risk factor and has been proposed, along with size and the presence of blebs, as the main aneurysm-specific characteristics to score aneurysm rupture risk. ${ }^{9}$ Our study suggests that location may be important because it may be associated with different local flow conditions - that is, adverse flow conditions may be more likely to de- 

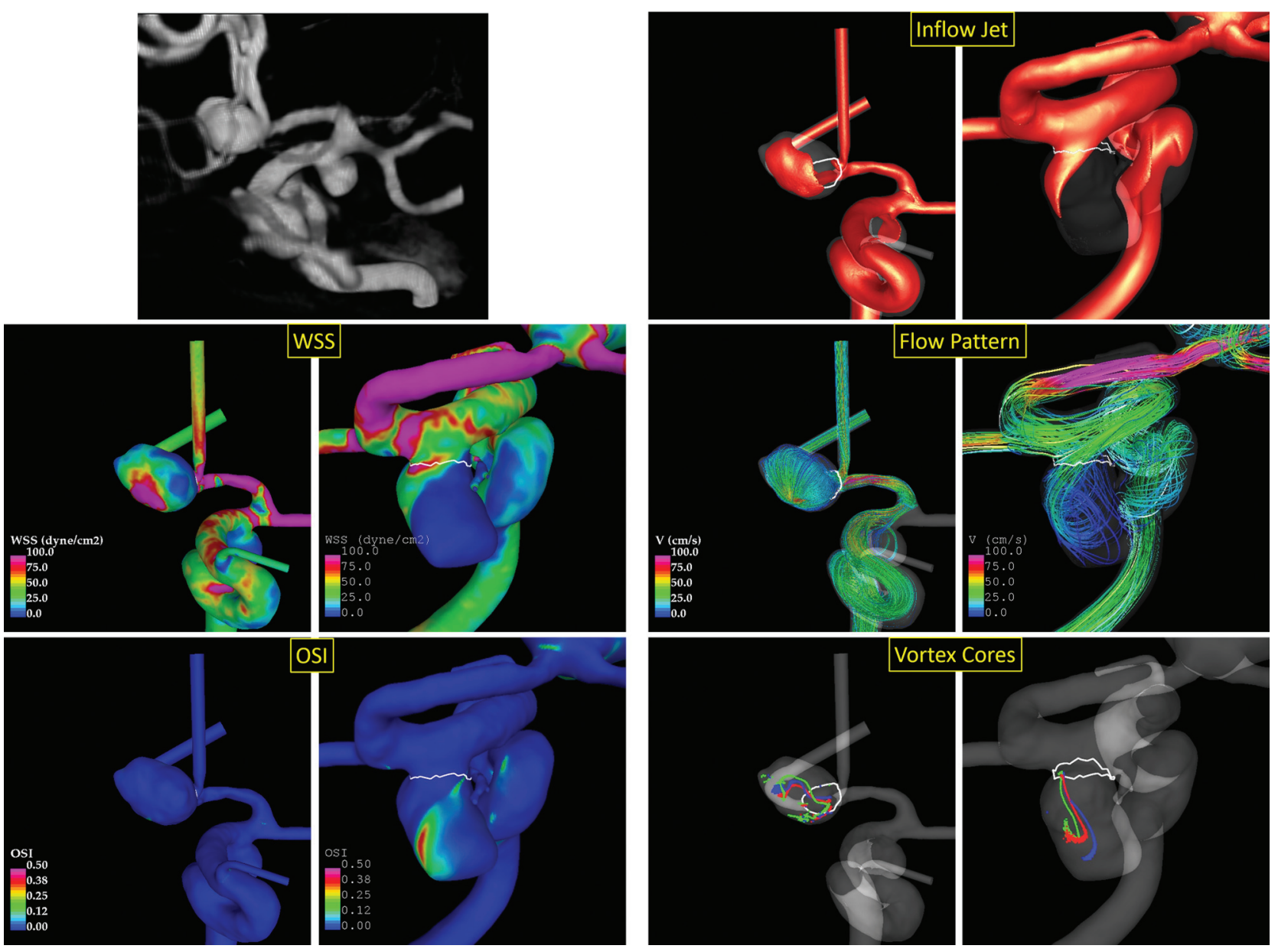

FIG 4. Example of ruptured (anterior communicating artery aneurysm fed from the left $\mathrm{Al}$ ) and unruptured (left posterior communicating artery aneurysm) multiple ipsilateral aneurysms. The left panel shows from top to bottom: 3D rotational angiography image, WSS distributions, and OSI distributions. The right panel shows from top to bottom: inflow jets, flow patterns, and vortex core lines at 4 time instances during the cardiac cycle.

velop at certain locations (for example more distally) and are less likely at other locations, independent of the flow rate in the feeding vessel. Furthermore, when we controlled for location as in the mirror-aneurysm analysis, fewer hemodynamic differences were observed.

Our study has some limitations. Although the sample size was large enough to achieve statistically significant results, it did not allow us to subdivide the sample to study bifurcation and sidewall aneurysms separately as has been suggested. ${ }^{33}$ Selection bias related to patient referral patterns and indications for treatment may have led to exclusion of important aneurysm subsets. The study was based on cross-sectional data; thus, it is not possible to determine whether the unruptured aneurysms had high or low rupture risk. Furthermore, the relative "ages" of the aneurysms in a single patient (ie, the time since they were formed) are not known, so it is not possible to determine their relative speed of progression. Finally, certain assumptions and approximations were made when constructing the computational fluid dynamics models, including assumptions of flow conditions, rigid walls, and Newtonian viscosity. Thus, the results should be confirmed with additional data from other populations, as well as with longitudinal data.

\section{CONCLUSIONS}

High wall shear stress oscillations and larger aspect ratios are associated with aneurysm rupture in mirror bilateral aneurysms. Hostile flow conditions characterized by high and concentrated inflow jets; high, concentrated, and oscillatory wall shear stress distributions; and strong, complex, and unstable flow patterns are associated with rupture in ipsilateral multiple aneurysms. These adverse flow conditions are more likely to develop in aneurysms that are more distal and larger and more elongated and have wider necks in multiple ipsilateral aneurysms.

Disclosures: Fernando Mut—RELATED: Grant: National Institutes of Health. ${ }^{*}$ Christopher M. Putman-UNRELATED: Payment for Lectures Including Service on Speakers Bureaus: Penumbra. Juan R. Cebral—RELATED: Grant: National Institutes of Health, Comments: research grant*; UNRELATED: Grants/Grants Pending: National Institutes of Health and Philips Healthcare, Comments: research grants. *Money paid to the institution.

\section{REFERENCES}

1. Juvela S, Porras M, Poussa K. Natural history of unruptured intracranial aneurysms: probability of and risk factors for aneurysm rupture. J Neurosurg 2008;108:1052-60 CrossRef Medline

2. Wiebers DO, Torner JC, Meissner I. Impact of unruptured intracra- 
nial aneurysms on public health in the United States. Stroke 1992; 23:1416-19 CrossRef Medline

3. Kelly PJ, Stein J, Shafqat $S$, et al. Functional recovery after rehabilitation for cerebellar stroke. Stroke 2001;32:530-34 Medline

4. Amenta PS, Yadla S, Campbell PG, et al. Analysis of nonmodifiable risk factors for intracranial aneurysm rupture in a large, retrospective cohort. Neurosurgery 2012;70:693-99; discussion 699-701 CrossRef Medline

5. Kaminogo M, Yonekura M, Shibata S. Incidence and outcome of multiple intracranial aneurysms in a defined population. Stroke 2003;34:16-21 CrossRef Medline

6. Baumann F, Khan N, Yonekawa Y. Patient and aneurysm characteristics in multiple intracranial aneurysms. Acta Neurchir Suppl 2008; 103:19-28 CrossRef Medline

7. Wang R, Zhang D, Zhao J, et al. A comparative study of 43 patients with mirror-like intracranial aneurysms: risk factors, treatment, and prognosis. Neuropsychiatr Dis Treat 2014;10:2231-27 CrossRef Medline

8. Ferguson GG. Physical factors in the initiation, growth, and rupture of human intracranial saccular aneurysms. J Neurosurg 1972;37: 666-77 CrossRef Medline

9. Greving JP, Wermer MJ, Brown RD Jr, et al. Development of the PHASES score for prediction of risk of rupture of intracranial aneurysms: a pooled analysis of six prospective cohort studies. Lancet Neurol 2014;13:59-66 CrossRef Medline

10. Lu HT, Tan HQ, Gu BX, et al. Risk factors for multiple intracranial aneurysms rupture: a retrospective study. Clin Neurol Neurosurg 2013;115:690-94 CrossRef Medline

11. Frösen J, Tulamo R, Paetau A, et al. Saccular intracranial aneurysm: pathology and mechanisms. Acta Neuropathol 2012;123:773-86 CrossRef Medline

12. Cebral JR, Castro MA, Appanaboyina S, et al. Efficient pipeline for image-based patient-specific analysis of cerebral aneurysm hemodynamics: technique and sensitivity. IEEE Trans Med Imag 2005;24:457-67 CrossRef Medline

13. Castro MA, Putman CM, Cebral JR. Patient-specific computational modeling of cerebral aneurysms with multiple avenues of flow from 3D rotational angiography images. Acad Radiol 2006;13: 811-21 CrossRef Medline

14. Mut F, Aubry R, Löhner R, et al. Fast numerical solutions of patientspecific blood flows in 3D arterial systems. Int J Num Meth Biomed Eng 2010;26:73-85 CrossRef Medline

15. Cebral JR, Castro MA, Putman CM, et al. Flow-area relationship in internal carotid and vertebral arteries. Physiol Meas 2008;29:585-94 CrossRef Medline

16. Mut F, Löhner R, Chien A, et al. Computational hemodynamics framework for the analysis of cerebral aneurysms. Int J Num Meth Biomed Eng 2011;27:822-39 CrossRef Medline

17. Byrne G, Mut F, Cebral JR. Quantifying the large-scale hemodynamics of intracanial aneurysms. AJNR Am J Neuroradiol 2014;35: 333-38 CrossRef Medline

18. Wachter D, Kreitschmann-Andermahr I, Gilsbach JM, et al. Early surgery of multiple versus single aneurysms after subarachnoid hemorrhage: an increased risk for cerebral vasospasm? J Neurosurg 2011;114:935-41 CrossRef Medline

19. Overgaard J, Riishede J. Multiple cerebral saccular aneurysms. Acta Neurol Scand 1966;41:363-71 Medline

20. Hoh BL, Sistrom CL, Firment CS, et al. Bottleneck factor and heightwidth ratio: association with ruptured aneurysms in patients with multiple cerebral aneurysms. Neurosurgery 2007;61:716-22; discussion 722-33 CrossRef Medline

21. Humphrey JD, Canham PB. Structure, mechanical properties, and mechanics of intracranial saccular aneurysms. Journal of Elasticity and the Physical Science of Solids 2000;61:49-81

22. Sforza DM, Putman CM, Cebral JR. Hemodynamics of cerebral aneurysms. Annu Rev Fluid Mech 2009;41:91-107 CrossRef Medline

23. Fan J, Wang Y, Liu J, et al. Morphological-hemodynamic characteristics of intracranial bifurcation mirror aneurysms. World Neurosurg 2015;84:114-20.e112 CrossRef Medline

24. Huang ZQ, Meng ZH, Hou ZJ, et al. Geometric parameter analysis of ruptured and unruptured aneurysms in patients with symmetric bilateral intracranial aneurysms: a multicenter CT angiography study. AJNR Am J Neuroradiol 2016;37:1413-17 CrossRef Medline

25. Zhang Y, Yang X, Wang Y, et al. Influence of morphology and hemodynamic factors on rupture of multiple intracranial aneurysms: matched-pairs of ruptured-unruptured aneurysms located unilaterally on the anterior circulation. BMC Neurol 2014;14:253 CrossRef Medline

26. Jou LD, Morsi H, Shaltoni HM, et al. Hemodynamics of small aneurysm pairs at the internal carotid artery. Med Eng Phys 2012;34: 1454-61 CrossRef Medline

27. Cebral J, Ollikainen E, Chung BJ, et al. Flow conditions in the intracranial aneurysm lumen are associated with inflammation and degenerative changes of the aneurysm wall. AJNR Am J Neuroradiol 2017;38:119-26 CrossRef Medline

28. Robertson AM, Duan X, Hill MR, et al. Diversity in the strength and structure of unruptured cerebral aneurysms. Ann Biomed Eng 2014; 43:1502-15 CrossRef Medline

29. Cebral JR, Duan X, Chung BJ, et al. Wall mechanical properties and hemodynamics of unruptured intracranial aneurysms. AJNR Am J Neuroradiol 2015;36:1695-703 CrossRef Medline

30. Cebral JR, Duan X, Gade PS, et al. Regional mapping of flow and wall characteristics of intracranial aneurysms. Ann Biomed Eng 2016;44: 3553-67 CrossRef Medline

31. Cebral JR, Mut F, Weir J, et al. Quantitative characterization of the hemodynamic environment in ruptured and unruptured brain aneurysms. AJNR Am J Neuroradiol 2011;32:145-51 CrossRef Medline

32. Xiang J, Natarajan SK, Tremmel M, et al. Hemodynamic-morphologic discriminants for intracranial aneurysm rupture. Stroke 2011; 42:144-52 CrossRef Medline

33. Baharoglu MI, Lauric A, Gao BL, et al. Identification of a dichotomy in morphological predictors of rupture status between sidewalland bifurcation-type intracranial aneurysms. J Neurosurg 2012;116: 871-81 CrossRef Medline 